\title{
Etiological Diagnosis of Fungal Keratitis and In Vitro Susceptibility Testing
}

\author{
Gong Xuewu,Wu Jing, Yao Hongbo \\ The Second Affiliated Hospital of Qiqihar Medical Colloge \\ Qiqihar, China
}

\begin{abstract}
Fungal keratitis is also known as the cornea fungal disease. Leber first reported in 1989. The disease is prevalent within a world-wide, more stubborn, which can be blinding fungal infection, it is often accompanied by corneal ulcers, purulent infection and impaired vision, severe corneal perforation can occur, endophthalmitis complications and even threatening the entire eye, causing blindness or loss of the affected eye, seriously affecting the health and quality of life of patients.
\end{abstract}

In recent years, due to the plant corneal trauma, chronic ocular surface diseases, eye surgery and corneal transplants have increased. The broad-spectrum antibiotics, corticosteroids abuse and improper use of contact lenses, as well as A worker DS, diabetes, systemic immune deficiency disease and other immunocompromised populations also increase. It is resulting in the incidence of fungal keratitis, fungal keratitis significantly increased incidence of occult infection progresses rapidly, early diagnosis is difficult; ophthalmology less common antifungal agents, and pathogenic fungi resistant serious difficulties effective treatment. Early diagnosis and prompt treatment is essential to control the infection. Therefore, the master of fungal keratitis law etiological and epidemiological characteristics, understand the diagnosis and treatment of common fungal infection, early and accurate diagnosis of the disease, timely and effective treatment and prognosis of recovery is important.

Keywords- Fungal Keratitis; Pathogen-shaped Diagnosis; Vitro Susceptibility Testing

\section{INTRODUCTION}

\section{A. Epidemiology law}

Fungal keratitis is common in developing countries in tropical and subtropical regions. In India, Thailand, Iran, Ghana, Brazil and other countries, the incidence rate of $30 \%$ to $50 \%$ of infectious keratitis. In China, more than 60 percent of serious infectious keratitis is caused by fungi. In contrast, in developed countries in temperate regions, especially the United States, the United Kingdom, the incidence of fungal keratitis is relatively low. It is reported that $6 \%$ to $53 \%$ of ulcerative keratitis is caused by fungal keratitis, most Asian countries blinding eye disease.

\section{B. Etiological features}

Currently, the cause of fungal keratitis or corneal infections other pathogenic fungi, 56 genera and 105 species of fungal keratitis pathogens can be classified according to their yeast and filamentous fungal keratitis. The former is the most common Candida albicans, as opportunistic pathogens, corneal ulcers caused by shallow, longer course, less likely to cause perforation. Whether the latter is common across the Zygomycetes such as Absidia and Rhizopus, and there is every other fungi, such as sophisticated Sydow Epidermophyton floccosum mildew, Fusarium, Aspergillus, bend hold mold, mildew hold the top branches, bottles mold, mildew and other chains holding cell. Among them, Fusarium and Aspergillus infection are the most common. Fusarium corneal ulcers caused severe symptoms, destructive strong, if not timely diagnosis and effective treatment can often lead to membrane perforation, or it can even lose the affected eye.

In recent years, the emergence of new infections in some plant pathogenic fungi or insects caused. Pariseau et al reported two cases of contact lens-related keratitis bassiana and Beauveria bassiana strain and biological pesticides random amplified polymorphic DNA (RAPD) analysis showed no correlation between the three strains. In 2010, Chew and other published reports from post-traumatic Ascomycota Sordariomycetes first cases of fungal keratitis.

Different climate, environment and other natural conditions are resulting in fungal keratitis pathogens differences among countries and regions. Tropical regions most isolated fungal keratitis and Fusarium fungi are Aspergillus, whereas in more temperate areas is common Fusarium and Candida. In India, it is causing fungal pathogen Aspergillus keratitis advantage, followed by bending mold hold. In Brazil, the most common is Fusarium, followed by Candida and Aspergillus. In the United States, more than half of the cases are caused by Candida.

\section{Pathological changes}

Features common fungal infection histopathological changes in the direct destruction of fungal corneal tissue stroma of normal structure is destroyed, a large number of inflammatory cells are to polymorph nuclear leukocytes mainly severe corneal epithelium and Descemet damage, leading to corneal ulceration and perforation. It is the Fungal-specific pathological changes of corneal tissue coagulation necrosis. This is due to pathogenic fungi corneal epithelial damage since enters stroma, the release of proteolytic enzymes, destruction of corneal tissue cells in the normal structure, the performance of coagulation necrosis, immune ring, endothelial plaque formation. Pathogenic fungi can proliferate within the matrix layer, extended depth, and then after Descemet penetrates into the anterior chamber. Studies have shown that Aspergillus fumigatus conidial can degrade laminin corneal layer. The toxins produced by fungi have certain invasive, which can lead to inflammatory cells, especially neutrophils accumulate a large number of the anterior chamber, the formation of hypopyon. The main pathological tissue damage is extensive fungal keratitis 
suppurative inflammation, corneal epithelial defect lesion, neutrophil infiltration around a large number of lesions, corneal stroma showed coagulation necrosis, swelling of collagen fibers between layers near the lesions may form a small abscess-like lesions, the latter course of polymorphonuclear leukocytes can be seen around the formation of chronic granulomatous fungal. PAS staining, the distribution layer of fibrous tissue around the lesion within the board a lot of fungal hyphae in the corneal stroma no law distribution, growth pattern may be parallel, oblique or perpendicular to the lamellar different pathogenic fungi and cornea stick attached to the role of different, different growth patterns and symptoms of infection, so that when the cause keratitis, corneal tissue injury are different.

Fungal damage morphology and development process by corneal disease can be divided into two types. Superficial lesions mainly located in the center or near the center of the cornea, less irritation, eye pain is not obvious, milder pathology, duration of up to several weeks to several months, more sensitive response to drug treatment. Visible lesion site lawn and surface uplift can occur early in the course of the cornea after the rear wall folds and calm things (inflammatory cells or pigmentation on the back surface of the cornea, KP), which may be associated with hypopyon, bundle-like lesions can be presented, dendritic, like other forms eroded. Deep in the type, it can affect the cornea deep, symptoms are abrupt, some were OK ulcer Austin appearance, suet-like KP, before

Housing empyema thick, iris, and conjunctivitis inflammatory reaction heavier ball are prone to corneal perforation. By pathogenic fungi grow in the corneal tissue; it can be divided into two types. Horizontal growth pattern, that is, the surface layer of carpet or plates grown mycelium in lesions around the horizontal or oblique spread hierarchical conduct infiltration, often associated with the inner layer of normal corneal tissue has obvious dividing line, smear-positive rate is higher, better, oblique growth, adapted to implement lamellar keratoplasty. Vertical growth pattern, a more serious infection, antifungal treatment hyphae vertically or deep stromal invasion and destruction of Descemet, clinical manifestations of early lesions smaller range, but the rapid onset, can form a significant endothelial plaque, satellite lesions, hypopyon, etc., due to poor permeability is often poor efficacy of antifungal agents, the short term can lead to corneal perforation, contraindications implement lamellar keratoplasty, penetrating keratoplasty good effect.

\section{MATERIALS AND METHODS ETIOLOGICAL DIAGNOSIS} OF FUNGAL KERATITIS

\section{A. Material}

January 1999 - December 20003, fungal smear results of a University Eye Center is a suppurative keratitis (except virus keratitis, exposure keratitis, etc.) specimens of 922 copies, fungal culture results of 1814 copies.

\section{B. Methods}

It is by professionals to operate. Specific methods of operation are with five-ophthalmology book. Check the fungal hyphae wet plate method using potassium hydroxide; fungal culture is using Sabouraud medium potatoes or potato dextrose medium. While conventional bacterial culture, the analysis and comparison of 1975 to 1986, from 1989 to 1997 in our hospital with suspected fungal keratitis patient laboratory data and related data analysis.

\section{Results}

Smear Results: From 1999 to 2003922 specimens' smears, 250 found fungal hyphae or spores, the positive rate of 27\%; of which 204 samples were positive fungal culture positive, smear and culture. Positive strains and fungal culture positive rate: 2010 - 2014 training for the clinical diagnosis of suppurative keratitis positive rate was $25 \%$. 1975 to 1986 culture-positive rate of $57.14 \%$; from 1989 to 1997 culture-positive rate of $52.3 \%$ (Table 2).

\section{Fungal culture-positive strains of seasonal distribution}

Our hospital from 1999 to 2003 has a total of 456 fungal culture, where the number of the onset of the first and second quarter of 142, the third and fourth quarter of 314 , the ratio between them is 1B212 (Figure 1). The main pathogenic fungi of the genus distribution: 1999 to 2003 a total of 456 fungal strains cultured, Fusarium 155 accounted for $34 \%$, Aspergillus 118 accounted for 26\%. Fusarium solani 107 $(69 \%)$ and Aspergillus fumigatus 59 (50\%) were the major pathogenic strains. In addition, there are 456 specimens of 80 parts bacterial culture positive, culture-positive bacterium Staphylococcus epidermidis accounted for $50 \%$. The main stages of pathogenic fungal strains and compared with the distribution of pre-hospital two stages shown in Figure 2.51 the last 40 years the incidence of fungal keratitis Review: In this hospital and diagnosed cases of fungal keratitis, in 1965 to 1970 an average of 3 cases / year, from 1970 to 1974 an average of 8 cases / year, from 1975 to 1980 an average of 18 cases / year. And since 1980, our hospital examination confirmed cases of fungal keratitis significantly increased: from 1981 to 1986 the average was 27 cases / year, from 1989 to 1997 and averages of 42 cases / year, from 1999 to 2003 an average of 91 cases / year.

\section{E. Discussion}

Currently, the laboratory diagnosis of fungal keratitis summed up the total stained smear cytology, biopsy, fungal culture, corneal confocal microscopy and polymerase chain reaction (PCR) technology. Among the many checks corneal scraping direct microscopic examination after staining method is still more valuable, can help confirm the diagnosis early. However, if a small number of fungi, hyphae smear layer is too thick or aging, the staining is often difficult to distinguish the mycelium state. Fungal culture takes a long time, it normally takes 2 to 19 days, but can be identified because of the strain, reflecting its morphological structure, and susceptibility testing, and thus fungal keratitis is 
important. Corneal Confocal microscopy shows significantly better than the other two methods. And has the advantages of the other two methods match. First, check the positive rate. Secondly, it is noninvasive without direct contact with the cornea lesions, without causing unnecessary damage to the cornea patients. Third, the rapid, early diagnosis, just a short time you can scan the full thickness corneal lesions.

\section{VITRO SUSCEPTIBILITY TESTING MATERIALS AND METHODS}

\section{A. Specimen source}

January 2006 to June 2007 was in a city eye hospital diagnosed cases of fungal keratitis. Laboratory and cultured corneal scraping by taking $10 \% \mathrm{KOH}$ dissolved after Giemsa staining find mycelium, while fungal culture medium by Showalter, after fungal colony morphology based on a combination of factors, mycelium, spore morphology identification. Production of fungal susceptibility testing kit for fungal susceptibility testing. Antifungal species in Table 1.

Table 1 types of antifungal drugs

\begin{tabular}{llccc}
\hline NO & antifungal & sensitive $(\mathrm{mg} / \mathrm{l})$ & intermediary $(\mathrm{mg} / \mathrm{l})$ & resistance $(\mathrm{mg} / \mathrm{l})$ \\
\hline 1 & Amphotericin $-\mathrm{B}$ & $\leqslant 1$ & 2 & $\geqslant 4$ \\
2 & Ketoconazole & $\leqslant 1$ & $2-8$ & $\geqslant 16$ \\
3 & Miconazole & $\leqslant 1$ & $2-4$ & $\geqslant 8$ \\
4 & Itraconazole & $\leqslant 0.12$ & $0.2-0.5$ & $\geqslant 1$ \\
5 & Fluconazole & $\leqslant 8$ & $16-32$ & $\geqslant 64$ \\
6 & Fluorocytosine & $\leqslant 4$ & $8-16$ & $\geqslant 2$ \\
7 & Terbinafine & $\leqslant 0.5$ & 1 & \\
\hline
\end{tabular}

\section{B. Results}

Species identification results: 96 positive cases, 64 cases of Fusarium; no Mortierella 13 cases; Alternaria spp nine cases; Aspergillus five cases; yeast three cases; Penicillium one case; Sporothrix schenckii 1 cases. Antimicrobial drug susceptibility testing are shown in Table 4.

\section{Discussion}

Fungal keratitis are deep mycosis, the incidence has obvious seasonal, our hospital between October to January fungal keratitis cases accounted for more than $65 \%$ of the year, while the incidence of trauma-related and more, especially plant trauma can easily lead to fungal infections. Diagnosis of fungal keratitis currently there are three: First, laboratory culture and smear slide method is to find fungal hyphae; the second is the corneal confocal microscopy; the third is the polymerase chain reaction (PCR) technology. Find smear laboratory fungal hyphae that is currently the more popular method, but due to the specimen sampling constraints, the positive rate is fixed and stained, and other aspects of the other methods is relatively low, can only reach about $55 \%$, as capable of This method is effective to improve, reduce intermediate links caused by misdiagnosis, for the early diagnosis of fungal keratitis is the most practical approach. Higher positive rate using laboratory specimens cultured, but need $2 \sim 7 \mathrm{~d}$, which is not conducive to early diagnosis and treatment of the disease. Corneal Confocal microscopy diagnosis rate of up to $85 \%$ more effective for the current method; PCR technology with a high positive rate compared slide method, the time is relatively rapid laboratory cultures, but there is the possibility of false positives, and it cannot be identified pathogenic bacteria, this method has not been widely used in clinical practice.

\section{CONCLUSIONS}

In short, summary, corneal confocal microscopy in the early diagnosis of fungal keratitis, treatment and has important clinical value study. But it can also pay attention to the corneal confocal microscopy by lesion size and depth of the impact on small lesions $(<1 \mathrm{~mm})$, surface repair deep unrepaired lesions cannot detect whether there exist good fungi. Its good imaging capabilities are closely with the patient's needs, any small eye movements will cause the image blurring, and therefore not suitable for children, patients with a high degree of disability and corneal perforation.

\section{ACKNOWLEDGEMENT}

Topic originates from the Qigihar Municipal Science and Technology Bureau

\section{REFERENCES}

[1] Sun Shiying. Fungal strains common analysis and in vitro drug sensitivity test [D]. Qingdao University, 2007.

[2] Qiao Yuanjiao. Diagnosis and treatment of fungal keratitis analysis [D]. Jilin University, 2009.

[3] Zhu Yefei. Fungal confocal microscopy diagnosis and susceptibility testing of clinically relevant research [D]. Zhejiang University, 2003.

[4] He Dan. Rapid detection of pathogens of fungal keratitis and major pathogen [D]. Jilin University, 2012.

[5] Yang Lianjuan, Xu Hong, Wen Hai. Laboratory diagnosis of fungal keratitis [J] Chinese Journal of fungi, 2007,02: 122-125.

[6] Zhao Lingjun. Analysis etiological features suppurative keratitis and infection risk factors [J] Recent Advances in Ophthalmology, 2014,09: 878-881. 